\title{
DISTÚRBIOS PSÍQUICOS MENORES (DPM) E QUALIDADE DE VIDA DOS ENFERMEIROS DO PROGRAMA DE SAÚDE DA FAMÍLIA DE FEIRA DE SANTANA, BAHIA.
}

\section{JAMILE PRADO OLIVEIRA SANTOS ${ }^{1}$; MÔNICA DE ANDRADE NASCIMENTO²; GABRIEL SILVA ROCHA ${ }^{3}$; KAROLE BRITO ALVES COSTA ${ }^{4}$; ROAN SILVA GOMES SAMAPAIO5.}

1. BOLSISTA PIBIC/FAPESB, GRADUANDA EM MEDICINA, UNIVERSIDADE ESTADUAL DE FEIRA DE SANTANA, E-MAIL: jamilleprado93@gmail.com

2. ORIENTADORA, DEPARTAMENTO DE SAÚDE, UNIVERSIDADE ESTADUAL DE FEIRA DE SANTANA, E-MAIL: andradenascimento.monica@gmail.com

3. BOLSISTA NA DE SITUAÇÃO E ANÁLISE EPIDEMIOLÓGICA E ESTATÍSTICA DO DEPARTAMENTO DE SAÚDE DA UNIVERSIDADE ESTADUAL DE FEIRA DE SANTANA, E-MAIL: gsrocha12@yahoo.com

4. BOLSISTA NA SALA DE SITUAÇÃO E ANÁLISE EPIDEMIOLÓGICA E ESTATÍSTICA DO DEPARTAMENTO DE SAÚDE DA UNIVERSIDADE ESTADUAL DE FEIRA DE SANTANA, E-MAIL: karolebrito@yahoo.com.br

5. BOLSISTA NA SALA de SITUAÇÃO E ANÁLISE EPIDEMIOLÓGICA E ESTATÍSTICA DO DEPARTAMENTO DE SAÚDE DA UNIVERSIDADE ESTADUAL DE FEIRA DE SANTANA, E-MAIL: roanrsgsampaio@gmail.com

PALAVRAS-CHAVE: PREVALÊNCIA; SRQ-20; PROGRAMA DE SAÚDE DA FAMÍLIA.

\section{INTRODUÇÃO}

O Programa Saúde da Família (PSF) foi criado em 1994 pelo Ministério da Saúde. Atualmente chamado Estratégia Saúde da Família (ESF), é um modelo prioritário para a reorganização da atenção básica à saúde no Brasil, configurando-se como porta de entrada ao Sistema Único de Saúde (SUS) (BRASIL, 1997). As mudanças e a precarização dos serviços de saúde, que podem gerar frustração, isolamento e submissão, e a falta de reconhecimento profissional, aliada à discussão tardia sobre planos de cargos e salários para os trabalhadores do SUS, ao arrocho salarial e ao aumento da demanda por serviços públicos de saúde, constituem-se em um estímulo à investigação das condições de trabalho dessas categorias profissionais, em especial entre aqueles que atuam na Estratégia Saúde da Família (BODSTEIN, 2002).

Distúrbio Psíquico Menor (DPM) é uma expressão criada por Goldberg \& Huxley (1993) para designar sintomas tais como insônia, fadiga, irritabilidade, dificuldade de concentração e de memorização, além de queixas somáticas, que sinalizam ruptura do funcionamento normal do indivíduo, mas não configuram categoria nosológica da $10^{\mathrm{a}}$ Classificação Internacional de Doenças (CID-10), bem como dos Manuais de Diagnóstico e Estatístico (DSM) da Associação Psiquiátrica Americana (COUTINHO ET AL., 1999). Entretanto, os distúrbios psíquicos menores constituem problema de saúde pública e 
apresentam impactos econômicos relevantes em função das demandas geradas aos serviços de saúde e do absenteísmo no trabalho (COUTINHO ET AL., 1999).

\section{MATERIAL E MÉTODOS}

O seguinte trabalho é um estudo epidemiológico populacional, de corte transversal, aplicado aos enfermeiros que atuam na Estratégia de Saúde da Família de Feira de Santana Bahia que consentirão em participar do estudo, após a leitura do Termo de Consentimento Livre e Esclarecido (TCLE). Para a coleta de dados foi utilizado um questionário padronizado, respondido pelos próprios profissionais, não sendo necessário que o mesmo se identifique. $\mathrm{O}$ questionário possui dois blocos de questões: $1^{\circ}$ bloco: identificação geral do entrevistado, destinado a caracterizar os indivíduos integrantes da amostra segundo sexo, idade tempo, carga horária total semanal, etc; $2^{\circ}$ bloco: contendo o SRQ-20 para detecção de DPM.

O SRQ foi desenvolvido por Harding et al. (1980) sob a coordenação da Organização Mundial de Saúde (OMS) com finalidade de estudar a morbidade psiquiátrica em instituições de saúde. A versão SRQ-20 é a mais utilizada em estudos de base populacional, sendo composta de 20 questões: 04 sobre sintomas físicos e 16 sobre sintomas psíquicos. As respostas são do tipo "sim" ou “não", atribuindo-se, respectivamente, valores de "1" e "0". O ponto de corte sugerido pelos autores para a suspeita de DPM é o de 07 respostas positivas. O trabalhador que apresentou escore $\geq 07$ respostas positivas foi considerado positivo ao SRQ20 .

A análise estatística dos dados foi realizada com uso do conjunto de programas SPSS for Windows da Sala de Situação e Análise Epidemiológica e Estatística do Departamento de Saúde da Universidade Estadual de Feira de Santana (SSAEE/DSAU/UEFS).

\section{RESULTADOS E DISCUSSÕES}

Participaram do estudo 71 enfermeiros que atuam na Estratégia de Saúde da Família no município de Feira de Santana, interior da Bahia. Entre os profissionais estudados 95,8 \% eram do sexo feminino, a maioria (77,1\%) tem mais de 32 anos e 50,8\% eram casados. Com relação ao vinculo de trabalho $97,1 \%$ eram cooperativados. A maioria $66,7 \%$ exerce uma carga horária semanal acima de 40 horas. 
Tabela 01. Perfil e características dos enfermeiros que trabalham na Estratégia de Saúde da Família em Feira de Santana-BA.

\begin{tabular}{l|c|c}
\hline VARIAVEIS & PERCENTUAL (\%) & ABSOLUTA \\
\hline SEXO & & \\
\hline FEMININO & 95,8 & 68 \\
MASCULINO & 4,2 & 03 \\
\hline IDADE & & 55 \\
\hline 32 ANOS & 77,1 & 16 \\
\hline < ANOS & 22,9 & \\
\hline ESTADO CIVIL & & 35 \\
\hline CASADOS & 50,8 & 27 \\
SOLTEIROS & 39,1 & 07 \\
OUTROS & 10,1 & 68 \\
\hline VÍNCULO DE TRABALHO & & 02 \\
\hline COOPERATIVADOS & 97,1 & \\
OUTROS & 2,9 & 40 \\
\hline CARGA HOÁRIA SEMANAL & & 22 \\
\hline <40 HORAS & 66,7 & \\
\hline 40 HORAS & 33,3 & \\
\hline
\end{tabular}

Acerca da prevalência de Distúrbio Psíquico Menor (DPM), encontra-se na Tabela 2 os valores determinados para o Questionário SRQ-20, indicando uma prevalência de DPM em $22,5 \%$ para o grupo de 71 validados.

Tabela 2. Índice de respostas positivas acima/ abaixo de 7 no questionário SRQ-20

\begin{tabular}{l|c|}
\hline \multicolumn{1}{|c|}{ SRQ-20 } & $\%$ \\
\hline NÚMERO DE RESPOSTAS POSITIVAS & \\
ACIMA DE 7 & 22,5 \\
\hline NÚMERO DE RESPOSTAS POSITIVAS & \\
ABAIXO DE 7 & 77,5 \\
\hline
\end{tabular}

Essa alta prevalência de DPM denota sério problema de saúde mental na população estudada. A prevalência encontrada foi superior a outros estudos de grupos ocupacionais como o estudo de trabalhadores de hospital, feito por Pitta (1990), o qual, identificou prevalência de 20,8\%, mas foi menor do que a encontrada por Rego (1992), que registrou prevalência global de 29\%. Já Araújo (1999) em estudo com profissionais da enfermagem e do sexo feminino encontrou uma prevalência de $33,3 \%$.

\section{CONCLUSÃO}


O presente estudo revela elevada prevalência de Distúrbio Psíquico Menor (DPM) entre enfermeiros do Programa de Saúde da Família em Feira de Santana com consequente redução da capacidade do indivíduo para o trabalho. Deve-se, então, re etir sobre que medidas podem ser adotadas para modificar as condições de trabalho, a relação prestador de serviços de saúde-paciente e a motivação desses profissionais, afinal, a Estratégia de Saúde de Família, além de ser a primeira via de promoção à saúde, é um ambiente desafiador, com os profissionais constantemente exposto a fatores estressantes. É somente por meio de estudos como este que é possível compreender os processos envolvidos no sofrimento psíquico desta categoria profissional e desenvolver intervenções mais eficazes, no sentido de melhorar a qualidade da saúde dos trabalhadores e consequentemente os serviços prestados aos usuários.

\section{REFERÊNCIAS}

ARAÚJO, TM. Distúrbios Psíquicos Menores entre mulheres trabalhadoras de enfermagem. [Tese de Doutorado]. Instituto de Saúde Coletiva da Universidade Federal da Bahia, Salvador, 1999.

BODSTEIN, R. Atenção básica na agenda da saúde. Ciências \& Saúde Coletiva, v. 7, n. 3, p. 401-412, 2002.

BRASIL. Ministério da Saúde. Saúde da família: uma estratégia para a reorientação do modelo assistencial. Brasília, DF, 1997.

BRASIL. Rev Psiquiatria Clínica; 26: 246-56.

COUTINHO, ESF; Almeida-Filho, N; Mari, JJ.1999. Fatores de risco para morbidade

GOLDBERG, D.; HUXLEY. 1992. P. Common mental disorders: a bio-social model. London: Tavistock Publications; New York: Routledge.

PITTA A. Hospital, 1990. Dor e morte como ofício. São Paulo:Hucitec. psiquiátrica menor: resultado de um estudo transversal em três áreas urbanas do

REGO MPCMA. 1992. Trabalho hospitalar e saúde mental: o caso de um hospital público no município do Rio de Janeiro [dissertação de mestrado]. Rio de Janeiro: Instituto de Medicina Social da UERJ. 